\title{
Isolated Pulmonary Presentation of Childhood Goodpasture's Disease
}

\author{
Ketan Kumar ${ }^{1}$, Joseph L. Mathew ${ }^{1}$, Lesa Dawman ${ }^{2}$, Ritambhra Nada ${ }^{2}$, PANKAJ \\ VAIDYA $^{2}$, and meenu singh ${ }^{3}$ \\ ${ }^{1}$ Postgraduate Institute of Medical Education and Research \\ ${ }^{2}$ PGIMER \\ ${ }^{3}$ Post Graduate Institute of Medical Education and Research,
}

September 11, 2020

\begin{abstract}
Antiglomerular basement membrane disease is a small vessel vasculitis which affects the lungs and kidneys, causing diffuse alveolar haemorrhage and rapidly progressive glomerulonephritis respectively. It is extremely uncommon in children. Diagnosis requires the presence of specific anti-GBM autoantibodies. Presentation with renal involvement is much more common than pulmonary involvement. However, a small proportion of cases might have isolated pulmonary involvement. Minimal changes on renal biopsy could still be present in these patients. Treatment of antiglomerular basement membrane disease includes plasmapheresis and immunosuppression. We present a case where a 7 year old boy presented with a long history suggestive of pulmonary haemorrhage but no renal manifestations and was diagnosed on the basis of anti-GBM autoantibodies and renal biopsy. He responded well to immunosuppressive therapy alone.
\end{abstract}

\begin{abstract}
Antiglomerular basement membrane disease is a small vessel vasculitis which affects the lungs and kidneys, causing diffuse alveolar haemorrhage and rapidly progressive glomerulonephritis respectively. It is extremely uncommon in children. Diagnosis requires the presence of specific anti-GBM autoantibodies. Presentation with renal involvement is much more common than pulmonary involvement. However, a small proportion of cases might have isolated pulmonary involvement. Minimal changes on renal biopsy culd still be present in these patients. Treatment of antglomerular basement membrane disease includes plasmapheresis and immunosuppression. We present a case where a 7 year old boy presented with a long history suggestive of pulmonary haemorrhage but no renal manifestations and was diagnosed on the basis of anti-GBM autoantibodies and renal biopsy. He responded well to immunosuppressive therapy alone.
\end{abstract}

To the Editor,

Goodpasture's disease or antiglomerular basement membrane (anti-GBM) disease is a small vessel vasculitis which presents as pulmonary-renal syndrome, causing rapidly progressive glomerulonephritis (RPGN) and diffuse alveolar haemorrhage (DAH). Goodpasture's syndrome is a non-specific term for any pulmonary-renal presentation, which is labelled as Goodpasture's disease in presence of anti-GBM autoantibodies. It has a bimodal age distribution and is rare in children ${ }^{1}$.

Majority of the patients present with RPGN, 40 to $60 \%$ of patients have simultaneous alveolar haemorrhage. Isolated pulmonary involvement at presentation is found in less than $10 \%$ patients. Diagnosis requires DAH and/or glomerulonephritis (GN) along with characteristic anti-GBM antibodies directed against the non-collagenous domain of $\alpha-3$ chain of type IV collagen, either in circulation or in tissue. Treatment 
usually involves plasmapheresis and immune-suppression and prognosis depends upon the extent of disease at diagnosis. We present a case of anti-GBM disease in a child who had long standing pulmonary involvement but no renal symptoms and was managed without plasmapheresis.

A 7 year old boy presented to our institution with history of recurrent episodes of pallor and generalized weakness, since the age of 3 years. He had previously received blood transfusion on four occasions. The lowest haemoglobin level $(\mathrm{Hb})$ documented was $3.5 \mathrm{~g} / \mathrm{dL}$. Most episodes were preceded by fever and dry cough but no breathlessness. In the most recent episode, he had been symptomatic for 3 months with intermittent fever, cough and 5-6 bouts of haemoptysis with small amounts of bright red blood. There was no history of dietary allergy, bleeding from any other site, haematuria or passage of cola coloured urine, rash, oral ulcers, joint or eye symptoms, or contact with active tuberculosis. On examination, growth was preserved, there was no hypertension, oedema or clubbing. There was mild pallor. Systemic examination including respiratory and cardiovascular systems was normal.

Hemoglobin was $8.4 \mathrm{~g} / \mathrm{dL}$ with hypochromia and microcytosis. Investigations confirmed iron deficiency. Chest xray from 1 year back showed bilateral infiltrates, predominantly involving right middle and lower and left lower zones (Figure 1A). However, fresh chest xray did not show these infiltrates. In view of chronic pulmonary symptoms with haemoptysis, iron deficiency anaemia and alveolar infiltrates on previous imaging, diffuse alveolar haemorrhage was considered.

CT angiography of the chest showed ill-defined alveolar opacities, consolidation and ground glassing involving the left upper lobe (Figure 1B). A flexible fibreoptic bronchoscopy was done under conscious sedation using oral triclofos and intravenous midazolam. No focal bleeding was observed. Bronchoalveolar lavage (BAL) analysis showed haemosiderin laden macrophages. Renal function tests were normal, urine microscopy done twice did not show any red blood cells and there was no proteinuria. Ophthalmic fundus examination did not show any changes suggestive of vasculitis. Serum ANA, ANCA and anti ds-DNA were negative. However, anti-GBM antibodies were positive $(25 \mathrm{IU} / \mathrm{ml})$. Therefore, a renal biopsy was done to look for glomerulonephritis associated with anti-GBM disease. It showed normal glomerular morphology on light microscopy (Figure 1C) on multiple serial sections, however direct immunofluorescence (repeated twice) showed 3+ linear positivity for IgG along the glomerular capillary walls (Figure 1D).

Within a few days, the child developed new onset fever, cough and haemoptysis. There was tachypnoea, tachycardia and hypoxia in room air. Auscultation revealed bilateral coarse crackles. Chest xray showed bilateral infiltrates. He received oxygen by nasal prongs and antibiotics, during which the symptoms resolved and xray infiltrates cleared. Again, there was no proteinuria or haematuria. The anti-GBM antibody level was $11 \mathrm{IU} / \mathrm{ml}$.

The child was administered methylprednisolone pulses for 3 days followed by oral prednisolone $(1 \mathrm{mg} / \mathrm{kg} / \mathrm{day})$ and cyclophosphamide $(2.5 \mathrm{mg} / \mathrm{kg} /$ day $)$. He was discharged on the same therapy and had no further episodes of haemoptysis or anaemia. Anti-GBM antibody levels done 2 months after starting therapy were negative $(2.6 \mathrm{IU} / \mathrm{ml})$. Follow-up over 6 months confirmed that the child remained well, with no requirement for further transfusions. He will continue to be closely monitored as the risk factors that determine prognosis in this particular subgroup of Goodpasture's disease have not been well studied.

Anti-GBM disease is extremely uncommon in children. In a retrospective review of more than 2000 renal biopsies and autopsies conducted over a period of 25 years at a tertiary centre in the USA, there were 4 cases of anti-GBM disease in children less than 18 years of age $^{2}$. Another review over a 30 year period identified a total of 24 cases younger than 16 years, with either Goodpasture's disease or syndrome ${ }^{3}$.

Haematuria, proteinuria and anaemia are the commonest symptoms in children with anti-GBM disease ${ }^{3}$. The unusual feature in the index case was the absence of symptoms or signs of renal dysfunction despite the long history. There are very few reports of children with only pulmonary symptoms. The terms 'Early Goodpasture syndrome' or 'Goodpasture variant' have been used for patients with pulmonary haemorrhage and anti-GBM antibodies without renal disease. Renal problems are said to develop within two to three years in most cases in this group. In these patients, light and electron microscopy of renal biopsy may be 
normal or show minimal changes ${ }^{4}$. It is unclear whether this group differs from that with renal presentation in terms of treatment and prognosis.

Most of the current treatment regimens for anti-GBM disease use plasmapheresis to rapidly remove circulating autoantibodies along with immune-suppression using cyclophosphamide and steroids. This is based on observational studies showing better outcomes with plasmapheresis ${ }^{1}$. The same studies reported using only steroids with cyclophosphamide in patients with lower antibody levels ${ }^{5}$. In our patient, the levels were not very high to start with. Therefore, we chose to go ahead with immunosuppression alone with close monitoring of the renal status.

Anti-GBM disease is a treatable condition and should be looked for in children with diffuse alveolar haemorrhage, even if renal involvement is not clinically apparent as happened in the index child. Factors predicting outcome include severity of renal dysfunction, requirement for dialysis, proportion of glomeruli with crescents and presence of interstitial infiltrate ${ }^{5}$. None of these risk factors were present in the index case.

\section{References}

1. Gulati K, McAdoo SP. Anti-Glomerular Basement Membrane Disease. Rheum Dis Clin North Am. 2018 Nov;44(4):651-673.

2. Williamson SR, Phillips CL, Andreoli SP, Nailescu C. A 25-year experience with pediatric anti-glomerular basement membrane disease. Pediatr Nephrol. 2011 Jan;26(1):85-91.

3. Bayat A, Kamperis K, Herlin T. Characteristics and outcome of Goodpasture's disease in children. Clin Rheumatol. 2012 Dec;31(12):1745-51.

4. Albelda SM, Gefter WB, Epstein DM, Miller WT. Diffuse pulmonary hemorrhage: a review and classification. Radiology. 1985 Feb;154(2):289-97.

5. Cui Z, Zhao J, Jia XY, Zhu SN, Jin QZ, Cheng XY, Zhao MH. Anti-glomerular basement membrane disease: outcomes of different therapeutic regimens in a large single-center Chinese cohort study. Medicine (Baltimore). 2011 Sep;90(5):303-11.

Figure 1. Panel A. Chest X ray showing bilateral diffuse fluffy infiltrates. Panel B. CT thoracic angiography showing left upper lobe consolidation and ground glassing. Panel C. Photomicrograph showing normal glomerulus which on immunofluorescence (Panel D) showed strong linear staining with IgG (Pas, FITC Tagged IgG, x 20 original magnification). 

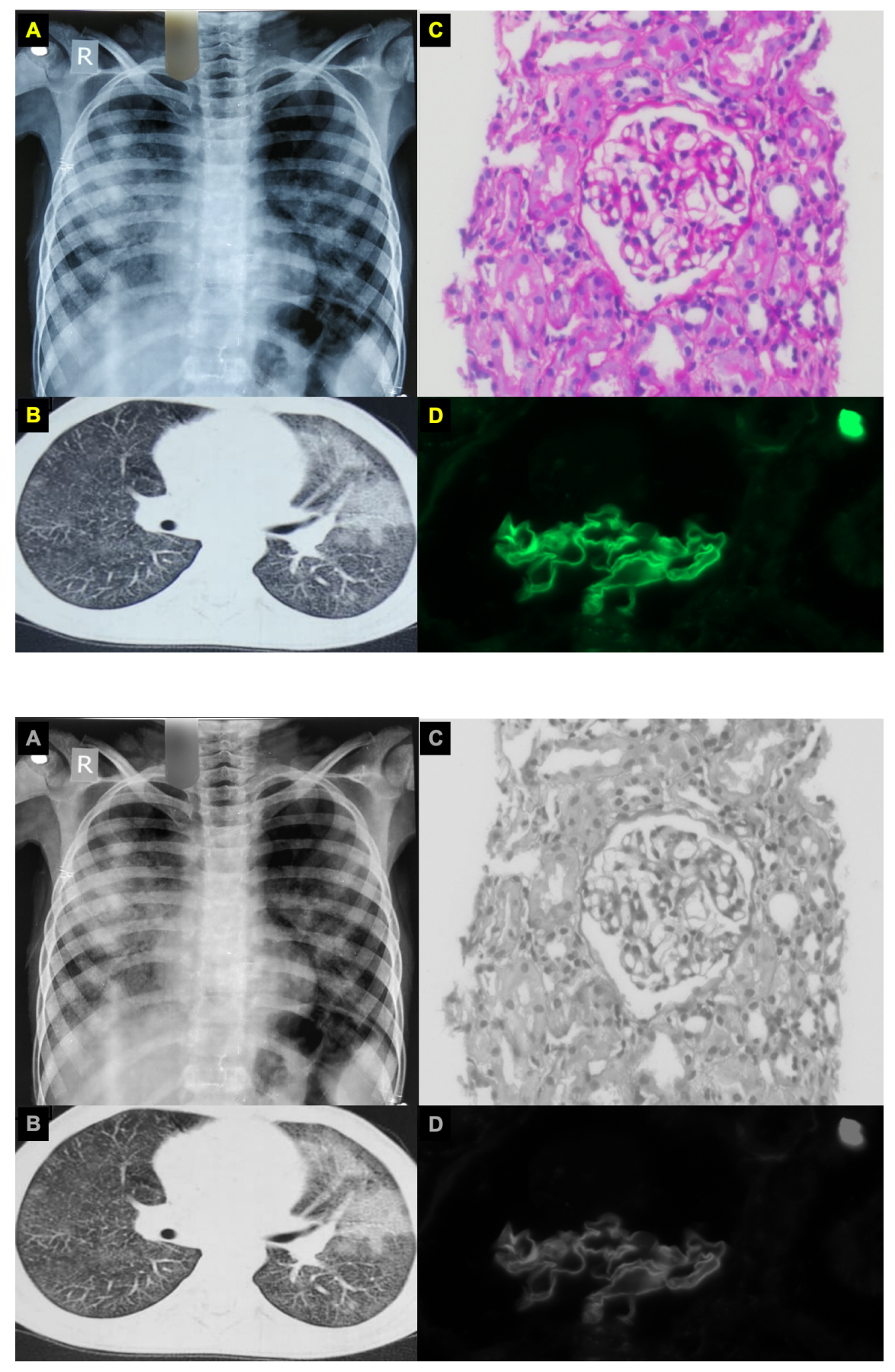